\title{
Indirect Dark Matter Search with the MAGIC Telescope
}

\author{
Adrian Biland*, Michael Rissi, Luisa Sabrina Stark Schneebeli \\ Institute for Particle Physics, ETH Zurich, CH-8093 Zurich, Switzerland \\ E-mail: biland@phys.ethz.ch, rissi@phys.ethz.ch, lstark@phys.ethz.ch
}

\section{Michele Doro, Saverio Lombardi}

Università di Padova and INFN, I-35131 Padova, Italy

E-mail: michele.doro@pd.infn.it, saverio.lombardi@pd.infn.it

\section{Francisco Prada, Miguel Sanchez-Conde, Fabio Zandanel}

Inst. de Astrofisica de Andalucia (CSIC), E-18080 Granada, Spain

E-mail: fprada@iaa.es, masc@iaa.es, fabio.zandanel@gmail.com

\section{Daniel Nieto}

Universidad Complutense, E-28040 Madrid, Spain

E-mail: nieto@gae.ucm.es

\section{for the MAGIC Collaboration}

http://wwwmagic.mppmu.mpg.de

The 17m Major Atmospheric Gamma-ray Imaging Cherenkov (MAGIC) Telescope located on the canary island La Palma has the lowest energy threshold of all existing Cherenkov Telescopes. This makes MAGIC well suited to look for high energy gamma rays coming from e.g. neutralino annihilation in Dark Matter (DM) dominated source candidates like spheroidal dwarf galaxies. So far, DRACO and Willman-I have been observed for several hours each, but no signal was found and the upper limits received look not very encouraging.

On the other hand, the AGILE and FERMI satellites might soon detect more promising candidates like hypothetical mini-halos or intermediate mass black holes. In such cases, follow up observations with Cherenkov telescopes with their much higher sensitivity above $\approx 100 \mathrm{GeV}$ will be needed to fully measure the spectra and search for a characteristic energy cutoff.

In case of a positive detection of a DM signal, an absolute energy calibration will be crucial. So far, Cherenkov telescopes had to rely fully on Monte Carlo simulations, resulting in a rather large systematic uncertainty. With an improved trigger electronics, MAGIC recently was able to see the cutoff energy of the Crab pulsar. Since this cutoff energy is within reach of FERMI, a cross-calibration of both instruments will soon be possible.

Identification of dark matter 2008

August 18-22, 2008

Stockholm, Sweden

${ }^{*}$ Speaker. 


\section{Introduction}

Many observations provide strong evidence for the existence of non-luminous, nonbarionic matter contributing about 6 times more to the total energy density of the universe than the baryonic matter described by the standard model of particle physics [1]. This so-called Dark Matter (DM) could be made of as yet unknown relic particles from the big bang, and seems to have only gravitational and probably weak interactions. Therefore, weakly interacting massive particles (WIMPs) are good candidates, with the lightest supersymmetric particle (neutralino) being one of the most favored. Stable neutralinos are predicted by many supersymmetric (SUSY) extensions of the standard model [2]. Such neutralinos would be Majorana particles and can therefore annihilate into standard model particles. The emission of gamma-rays would be most interesting, since they travel through the universe without being affected by the omnipresent magnetic fields and can be traced back to their origins. Direct annihilation into $\gamma \gamma$ or $\gamma Z$ would produce sharp line signals with a photon energy directly related to the neutralino mass. But such processes are loop suppressed and therefore very rare. Far more abundant are annihilations into quark or $\tau$ pairs, that in subsequent standard model processes produce $\gamma$-rays via $\pi^{0}$ decays. The spectrum of these photons will be continuous with a hard cutoff at the neutralino mass. The majority of the $\gamma$-rays would originate from annihilations into quarks, but those coming from $\tau$ decays have in average much higher energies and are therefore easier to detect with Imaging Air Cherenkov Telescopes (IACT).

\section{The MAGIC Telescope and Standard Analysis}

The MAGIC Telescope is located at the Roque de los Muchachos Observatory on the canary island La Palma at an altitude of about $2200 \mathrm{~m}$ a.s.l. Thanks to its large $17 \mathrm{~m}$ tessellated reflector dish, it reaches the lowest energy threshold $(\approx 55 \mathrm{GeV}$ at the trigger level for small zenith angles) of all current IACTs. The accessible energy range extends up to tens of $\mathrm{TeV}$ with a typical energy dependent energy resolution of $20 \%-30 \%$ [3].

The field of view of the 576 pixel photomultiplier camera is $3.5^{\circ}$, limited by aberration effects induced by the parabolic shape of the reflector dish. The angular resolution is $\approx 0.1^{\circ}$, mainly limited by statistical fluctuations in the shower development.

The standard calibration and analysis is based on image parameters [4][5] and the random forest method [6] to define the so-called hadronness of each event. The cut in this hadronness parameter for the crucial $\gamma /$ hadron separation is optimized on contemporaneous data sets from well known sources like the Pulsar Wind Nebula around the Crab pulsar as well as comparing with Monte Carlo simulations of air showers [7]. After this cut the distribution of the angle ALPHA, which is the angle between the main image axis and the line connecting the center of gravity of the image and the source position in the camera is used to determine the signal in the ON-source region. The remaining background can be determined by two different methods: in the ON-OFF mode, intermittent observations of a point in the sky with similar observation conditions but no expected gamma-ray source are made, while for the wobble mode [8] the observation time is split between the telescope pointing $0.4^{\circ}$ off the source in one direction, and its mirror point.

The energy of each $\gamma$-ray candidate is also estimated by the random forest method, using Monte Carlo events as training sample. In case of a positive signal from a source, the derived spectrum 
has to be unfolded to correct for effects of limited energy resolution [9]. Additionally, at least two independent analysis have to agree within expected errors before a detection can be claimed.

\section{Source Candidates}

The detectable $\gamma$-ray flux originating from e.g. neutralino annihilation at a hypothetical source depends on three main factors. While the first part, sensitivity and energy coverage of the detector are known precisely, this is not the case for the other ingredients. Particle Physics theories predict cross-section as well as spectrum and number of $\gamma$-rays produced per annihilation, but depending on the exact theory and region in the vast parameter space, these predictions can differ by several orders of magnitude. Finally, the annihilation rate depends on the squared density of the DM distribution along the line of sight. The average DM density distribution is not known very well, and there exists also the possibility of rather large boost factors originating from e.g. possible clumpiness.

Since the Particle Physics part is independent of the source, best candidates to look at are nearby regions with expected high DM density. Probably the most favorable source would be the center of our galaxy, but recent measurements by H.E.S.S. [10], confirmed by MAGIC [11], proof the existence of a bright $\gamma$-ray source within the field of view obscuring any possible signal coming from DM annihilation processes.

Also nearby galaxies are no ideal candidates because they too are expected to contain several bright astrophysical $\gamma$-ray emitters. Better objects could be galaxy clusters, but the limited field of view and angular resolution of IACTs makes them rather difficult to observe.

Other candidates would be hypothetical, nearby DM clumps like mini-halos [12][13] or Intermediate Mass Black Holes [14]. But since such sources would only be visible by $\gamma$-rays originating from DM annihilations, their positions are unknown. Because of the small field of view, IACTs are not suited to scan large areas of the sky, and such sources could only be serendipity detections while observing other targets.

This leaves as candidates the spheroidal dwarf galaxies in close vicinity of our own galaxy. From astrophysical observations it is known they have a very high Mass/Luminosity ratio and therefore are expected to have a high DM density.

\section{Results}

In 2007 and 2008 MAGIC observed the spheroidal dwarf galaxies DRACO [15][16] and Willman-1 [17] for 7.8 and 15.5 hours respectively. For DRACO, a flux upper limit above $140 \mathrm{GeV}$ of $\approx 10^{-11} \gamma$-rays cm $\mathrm{cm}^{-2} \mathrm{~s}^{-1}$ was found [18], while for Willman-1 the limit above $100 \mathrm{GeV}$ is about one order of magnitude better [19]. Taking reasonable assumptions for the DM density distributions, and using the benchmark models defined in [20] to describe the neutralino, it is only possible to rule out huge boost factors of $\leq 6.5 \times 10^{3}$ for DRACO and $\leq 2.9 \times 10^{3}$ for Willman- 1 for the model K', predicting the highest $\gamma$-ray flux $\left(m_{1 / 2}=1300, m_{0}=1001, \tan \beta=46, A_{0}=0, \mu=-1\right)$. 


\section{Outlook}

While these results look not very encouraging, it has to be noted that $\gamma$-ray observations can give much more information about DM in the universe than the detection of a DM-candidate particle at LHC, a positive signal in direct search experiments or an excess of anti-p or $e^{+}$in cosmic ray experiments. $\gamma$-rays have the potential to investigate DM distribution as well as intrinsic properties of a DM building particle like its mass.

Recent realization that so far overlooked QED corrections in DM annihilation processes can result in significantly enhanced $\gamma$-ray emission, especially at the highest energies [21], is a very interesting development. These processes are included in the latest version of the DarkSusy simulation program [22]. Unfortunately, several of the standard benchmark models become incompatible with the WMAP constraints when recalculated with the new program. Additionally, these benchmark models have all been defined with $A_{0}=0$, while non-vanishing trilinear coupling can result in enhanced t-channel branching ratio and thus in possibly enlarged QED correction effects. Therefore new scans of e.g. the mSUGRA phase-space as well as definitions of new benchmark points would be rather important for the whole community.

The sensitivity of several IACTs is currently improved, e.g. a second MAGIC telescope will start taking data in 2009. By observing the identical air showers with both telescopes in stereo mode, it will be possible to do a better $\gamma /$ hadron separation and therefore significantly improve the sensitivity, especially at low energies. Additionally, the design of next generation Cherenkov observatories has already started, and with an expected 10-fold increase in sensitivity, a DM signal from several sources might come into reach [23].

Last but not least, the recently launched FERMI satellite (formerly known as GLAST) with its full-sky coverage might soon find some unidentified sources with hard spectra that could be hypothetical DM clumps. Follow-up observations with IACTs and their much higher sensitivity above $\approx 100 \mathrm{GeV}$ will be very important, and cooperation between FERMI and IACT groups is already established.

Aside of the low energy threshold, there is another reason making MAGIC an ideal instrument for follow-up observations of unidentified FERMI sources: with a modified trigger electronics, it was possible to reach a trigger threshold of $\approx 25 \mathrm{GeV}$ and therefore to measure for the first time the cutoff energy of pulsed emission from the Crab pulsar $[24]^{1}$. Since this very sharp cutoff is in the energy range of FERMI, it will soon be possible to use it to cross-calibrate the two instruments and therefore for the first time get an absolute energy calibration of an IACT. This is very important in case of the detection of $\gamma$-rays from DM annihilation, since the cutoff energy is of uttermost importance for understanding the DM.

\section{References}

[1] D. N. Spergel et al., (WMAP Collab), Three-Year Wilkinson Microwave Anisotropy Probe (WMAP) Observations: Implications for Cosmology, Astrop. J, 170, 377 (2007)

[2] G. Jungman \& M. Kamionkowsky, $\gamma$-rays from neutralino annihilation, Phys. Rev. D51 (1995)

\footnotetext{
${ }^{1}$ It has to be noted that the $\gamma$-hadron separation is very difficult at such low energies and it can not be expected MAGIC to be able to measure regularly faint sources at such low energies in near future.
} 
[3] J. Albert et al. (MAGIC Collab), VHE Gamma-Ray Observation of the Crab Nebula and Pulsar with MAGIC, Astrophys. J. 674, 1037 (2008)

[4] A. M. Hillas, Cherenkov light images of EAS produced by primary gamma rays and by nuclei, Proc. 19th Int. Cosm. Ray Conf., La Jolla, 1985

[5] Tescaro et al. (MAGIC Collab) Study of the performance and capability of the new ultra-fast 2 GSample/s FADC data acquisition system of the MAGIC telescope, Proc. 30th Int. Cosm. Ray Conf., Merida, 2007, arXiv:0709.1410

[6] J. Albert et al. (MAGIC Collab), Implementation of the Random Forest Method for the Imaging Atmospheric Cherenkov Telescope MAGIC, Nucl. Instr. Meth. A 588, 424 (2008)

[7] D. Heck et al., CORSIKA: A Monte Carlo to Simulate Extensive Air Showers, Karlsruhe, http://www-ik.fzk.de/corsika/

[8] A. Daum et al. (HEGRA Collab), First results on the performance of the HEGRA IACT array, Aatropart. Phys, 8, 1 (1997)

[9] J. Albert et al. (MAGIC Collab), Unfolding of differential energy spectra in the MAGIC experiment, Nucl. Instr. Meth. A 583, 494 (2007)

[10] F. Aharonian et al. (H.E.S.S. Collab), Very high energy gamma rays from the direction of Sagittarius $A^{*}$, Astron. \& Astrophys. 425, L13 (2004)

[11] J. Albert et al. (MAGIC Collab), Observation of Gamma Rays from the Galactic Center with the MAGIC telescope, Astrophys. J. 638, L101 (2006)

[12] J. Diemand et al., Formation and Evolution of Galaxy Dark Matter Halos and Their Substructure Astrophys. J. 667, (2007)

[13] L. Strigari et al., Redefining the Missing Satellites Problem Astrophys. J. 669, (2007)

[14] G. Bertone et al., New signature of dark matter annihilations: Gamma rays from intermediate-mass black holes, Phys. Rev. D72 (2005)

[15] L. Bergström \& D. Hooper, Dark matter and gamma rays from Draco: MAGIC, GLAST and CACTUS, Phys. Rev. D73 (2006)

[16] L. Mayer et al., Early gas stripping as the origin of the darkest galaxies in the Universe, Nature, 445 (2007)

[17] B. Willman et al., A New Milky Way Dwarf Galaxy in Ursa Major, Astrop. J. 626 L85 (2005)

[18] J. Albert et al., (MAGIC Collab), Upper limit for gamma-ray emission above 140 GeV from the dwarf spheroidal galaxy Draco, Astrophys. J. 679, 428 (2008)

[19] E. Aliu et al., (MAGIC Collab), MAGIC upper limits on the VHE gamma-ray emission from the satellite galaxy Willman-1, submitted 2008, arXiv:0810.3561

[20] M. Battaglia et al., Updated post-WMAP benchmarks for supersymmetry, European Phys. J. C, 33, 273 (2004)

[21] T. Bringman, L. Bergstöm and J. Edsjö, New Gamma-Ray Contributions to Supersymmetric Dark Matter Annihilation, JHEP 801, 049 (2008)

[22] P. Gondolo, J. Edsjö, P. Ullio, L. Bergström, M. Schelke and E.A. Baltz, DarkSUSY: computing supersymmetric dark matter properties numerically, JCAP 0407 (2004) 008

[23] T. Bringman, M. Doro and M. Fornasa, Dark Matter signals from Draco and Willman 1: Prospects for MAGIC II and CTA, accepted by JCAP 2008, arXiv:0809.2269

[24] E. Aliu et al., (MAGIC Collab), Observation of Pulsed $\gamma$-Rays Above 25 GeV From the Crab Pulsar with MAGIC, accepted by Science 2008, http://www.sciencemag.org/cgi/content/abstract/1164718 